\title{
A!
}

This is an electronic reprint of the original article.

This reprint may differ from the original in pagination and typographic detail.

Heikkinen, Toni; Kinnunen, Juha

\section{A median approach to differentiation bases}

Published in:

RENDICONTI LINCEI: MATEMATICA E APPLICAZIONI

DOI:

10.4171/RLM/835

Published: 01/01/2019

Document Version

Peer reviewed version

Please cite the original version:

Heikkinen, T., \& Kinnunen, J. (2019). A median approach to differentiation bases. RENDICONTI LINCEI:

MATEMATICA E APPLICAZIONI, 3O(1), 41-66. https://doi.org/10.4171/RLM/835

This material is protected by copyright and other intellectual property rights, and duplication or sale of all or part of any of the repository collections is not permitted, except that material may be duplicated by you for your research use or educational purposes in electronic or print form. You must obtain permission for any other use. Electronic or print copies may not be offered, whether for sale or otherwise to anyone who is not an authorised user. 


\title{
A MEDIAN APPROACH TO DIFFERENTIATION BASES
}

\author{
TONI HEIKKINEN AND JUHA KINNUNEN
}

\begin{abstract}
We study a version of the Lebesgue differentiation theorem in which the integral averages are replaced with medians over Busemann-Feller differentiation bases. Our main result gives several characterizations for the differentiation property in terms of the corresponding median maximal function. As an application, we study pointwise behaviour in Besov and Triebel-Lizorkin spaces, where functions are not necessarily locally integrable. Most of our results apply also for functions defined on metric measure spaces.
\end{abstract}

\section{INTRODUCTION}

If $f$ is a locally integrable function, then by the classical Lebesgue differentiation theorem,

$$
\lim _{r \rightarrow 0} \frac{1}{|B(x, r)|} \int_{B(x, r)} f(y) d y=f(x)
$$

for almost every $x \in \mathbb{R}^{n}$. Here, $B(x, r)=\left\{y \in \mathbb{R}^{n}:|x-y|<r\right\}$ is an open ball of radius $r>0$ centered at $x$ and $|A|$ denotes the Lebesgue measure of set $A \subset \mathbb{R}^{n}$. If the integral averages in (1.1) are replaced by medians, or more generally, by $\gamma$-medians,

$$
m_{f}^{\gamma}(A)=\inf \{a \in \mathbb{R}:|\{x \in A: f(x)>a\}|<\gamma|A|\},
$$

where $A \subset \mathbb{R}^{n}$ is a bounded and measurable set and $0<\gamma<1$, then for every measurable function $f: \mathbb{R}^{n} \rightarrow[-\infty, \infty]$, with $|f(x)|<\infty$ for almost every $x \in \mathbb{R}^{n}$, we have

$$
\lim _{r \rightarrow 0} m_{f}^{\gamma}(B(x, r))=f(x)
$$

for almost every every $x \in \mathbb{R}^{n}$, see [4], [28]. Ler $L^{0}\left(\mathbb{R}^{n}\right)$ denote the set of all measurable functions $f: \mathbb{R}^{n} \rightarrow[-\infty, \infty]$ such that $|f(x)|<\infty$ for almost every $x \in \mathbb{R}^{n}$. Thus the Lebesgue differentiation theorem with medians holds for functions in $L^{0}\left(\mathbb{R}^{n}\right)$.

It is natural to ask whether (1.1) or (1.2) still hold true if the balls are replaced by some other collections of sets converging to $x$. In the case of integral averages, this problem has been studied by many authors, for example, see [1], [2] and the references therein. It is known that if $\mathcal{B}$ is a homothecy invariant Busemann-Feller basis (see Definition 2.2), then for every $f \in L^{1}\left(\mathbb{R}^{n}\right)$, we have

$$
\lim _{\mathcal{B} \ni B \rightarrow x} \frac{1}{|B|} \int_{B} f(y) d y=f(x)
$$

2010 Mathematics Subject Classification. 42B25, 46E35. 
for almost every $x \in \mathbb{R}^{n}$ if and only if there exists a constant $C$ such that the corresponding maximal function

$$
\mathcal{M}_{\mathcal{B}} f(x)=\sup _{x \in B \in \mathcal{B}} \frac{1}{|B|} \int_{B}|f(y)| d y
$$

satisfies the weak type estimate

$$
\left|\left\{x \in \mathbb{R}^{n}: \mathcal{M}_{\mathcal{B}} f(x)>\lambda\right\}\right| \leq C \lambda^{-1}\|f\|_{L^{1}\left(\mathbb{R}^{n}\right)}
$$

for every $f \in L^{1}\left(\mathbb{R}^{n}\right)$ and $\lambda>0$. A striking fact is that the qualitative Lebesgue differentiation theorem is characterized through a quantitative weak type estimate for the corresponding maximal function.

The purpose of this note is to obtain similar results for medians and the median maximal function

$$
\mathcal{M}_{\mathcal{B}}^{\gamma} f(x)=\sup _{x \in B \in \mathcal{B}} m_{|f|}^{\gamma}(B)
$$

where $f: \mathbb{R}^{n} \rightarrow[-\infty, \infty]$ is a measurable function with $|f(x)|<\infty$ for almost every $x \in \mathbb{R}^{n}$. Medians and related maximal functions have turned out to be useful in harmonic analysis and function spaces, see [3], [4], [5], [8], [9], [11], [13], [14], [15], [16], [17], [18], [24], [26], [27], [28], [30], [31], [33]. The main advantage of a median over an integral average is that it applies also when the function is not necessarily locally integrable. This is relevant in certain function spaces, where functions are not necessarily locally integrable and thus integral averages are not defined. As we shall see, in many cases medians are more tractable than integral averages.

For homothecy invariant Busemann-Feller bases, we will prove the following theorem.

Theorem 1.1. Let $\mathcal{B}$ be a homothecy invariant Busemann-Feller basis on $\mathbb{R}^{n}$. Then the following claims are equivalent.

(1) $\mathcal{B}$ is a density basis, that is, for every measurable $A \subset \mathbb{R}^{n}$,

$$
\lim _{\mathcal{B} \ni B \rightarrow x} \frac{|A \cap B|}{|B|}=\chi_{A}(x)
$$

for almost every $x \in \mathbb{R}^{n}$.

(2) For every $0<\gamma<1$ and $f \in L^{0}\left(\mathbb{R}^{n}\right)$,

$$
\lim _{\mathcal{B} \ni B \rightarrow x} m_{f}^{\gamma}(B)=f(x)
$$

for almost every $x \in \mathbb{R}^{n}$.

(3) For every $0<\gamma<1$, there exists a constant $C$ such that

$$
\left|\left\{x \in \mathbb{R}^{n}: \mathcal{M}_{\mathcal{B}}^{\gamma} f(x)>\lambda\right\}\right| \leq C\left|\left\{x \in \mathbb{R}^{n}:|f(x)|>\lambda\right\}\right|
$$

for every $\lambda>0$ and $f \in L^{0}\left(\mathbb{R}^{n}\right)$.

(4) For every $0<\gamma<1$ and $0<p<\infty$, there exists a constant $C$ such that

$$
\left\|\mathcal{M}_{\mathcal{B}}^{\gamma} f\right\|_{L^{p}\left(\mathbb{R}^{n}\right)} \leq C\|f\|_{L^{p}\left(\mathbb{R}^{n}\right)}
$$

for every $f \in L^{p}\left(\mathbb{R}^{n}\right)$. 
(5) There exists $0<p<\infty$ such that, for every $0<\gamma<1, \lambda>0$ and for every sequence $\left(f_{k}\right)_{k \in \mathbb{N}}$ such that $\left\|f_{k}\right\|_{L^{p}\left(\mathbb{R}^{n}\right)} \rightarrow 0$ as $k \rightarrow \infty$, we have

$$
\left|\left\{x \in \mathbb{R}^{n}: \mathcal{M}_{\mathcal{B}}^{\gamma} f_{k}(B)>\lambda\right\}\right| \rightarrow 0
$$

as $k \rightarrow \infty$.

(6) There exists $0<p<\infty$ such that, for every $0<\gamma<1$ and $f \in L^{p}\left(\mathbb{R}^{n}\right)$,

$$
\mathcal{M}_{\mathcal{B}}^{\gamma} f(x)<\infty
$$

for almost every $x \in X$.

Homothecy invariance and special covering properties of the Euclidean spaces are needed only in showing that (1) implies (3). Assertions (1), (2), (5) and (6) are equivalent in more general metric measure spaces.

As an application, we study pointwise behaviour of functions in Besov and TriebelLizorkin spaces. We employ the definitions of Besov and Triebel-Lizorkin spaces in metric measure spaces introduced in [23]. This definition is motivated by the Hajłasz-Sobolev spaces, see [6] and for fractional scales [32]. The functions in these spaces are more regular than arbitrary measurable functions, but they are not necessarily locally integrable. Exceptional sets are measured with the corresponding capacity instead of the underlying measure. We give several characterizations of (1.3) in the context of metric measure spaces. The results are new already in the Euclidean case with Lebesgue measure, but definitions of the function spaces in more general metric measure spaces give a transparent and flexible approach to pointwise behaviour.

\section{Preliminaries}

2.1. Basic assumptions. In this paper, $X=(X, d, \mu)$ denotes a metric measure space equipped with a metric $d$ and a Borel regular outer measure $\mu$, for which the measure of every ball is positive and finite.A measure $\mu$ is doubling if there exists a constant $C_{d}$, such that

$$
\mu(B(x, 2 r)) \leq C_{d} \mu(B(x, r))
$$

for every ball $B(x, r)=\{y \in X: d(y, x)<r\}$, where $x \in X$ and $r>0$. The doubling condition is equivalent to existence of constants $C$ and $Q$ such that

$$
\frac{\mu(B(y, r))}{\mu(B(x, R))} \geq C\left(\frac{r}{R}\right)^{Q}
$$

for every $0<r \leq R$ and $y \in B(x, R)$.

The integral average of a locally integrable function $f$ over a measurable set $A$ of positive and finite measure is denoted by

$$
f_{A}=f_{A} f d \mu=\frac{1}{\mu(A)} \int_{A} f d \mu .
$$

The characteristic function of a set $E \subset X$ is denoted by $\chi_{E} . L^{0}(X)$ is the set of all measurable functions $f: X \rightarrow[-\infty, \infty]$ such that $|f(x)|<\infty$ for almost every $x \in X$. In general, $C$ denotes a positive and finite constant whose value are not 
necessarily same at each occurrence. When we want to emphasize that the constant depends on parameters $a, b, \ldots$, we write $C=C(a, b, \ldots)$.

2.2. $\gamma$-median. Let $A \subset X$ be a measurable set with $\mu(A)<\infty$. For $0<\gamma<1$, the $\gamma$-median of a measurable function $f: X \rightarrow[-\infty, \infty]$ over $A$ is

$$
m_{f}^{\gamma}(A)=\inf \{a \in \mathbb{R}: \mu(\{x \in A: f(x)>a\})<\gamma \mu(A)\} .
$$

Note that $m_{f}^{\gamma}(A)$ is finite, if $f \in L^{0}(A)$ and $0<\mu(A)<\infty$. We list some basic properties of the $\gamma$-median below. Properties $(1),(2),(4),(6),(7)$ and (8) are proved in the Euclidean setting in [28, Propositions 1.1 and 1.2]. The remaining properties follow immediately from the definition.

Lemma 2.1. Let $A, B \subset X$ be measurable sets with $\mu(A)<\infty$ and $\mu(B)<\infty$ and assume that $f, g: X \rightarrow[-\infty, \infty]$ are measurable functions.

(1) If $0<\gamma \leq \gamma^{\prime}<1$, then $m_{f}^{\gamma}(A) \geq m_{f}^{\gamma^{\prime}}(A)$.

(2) If $f \leq g$ almost everywhere in $A$, then $m_{f}^{\gamma}(A) \leq m_{g}^{\gamma}(A)$.

(3) If $A \subset B$ and $\mu(B) \leq C \mu(A)$, then $m_{f}^{\gamma}(A) \leq m_{f}^{\gamma / C}(B)$.

(4) $m_{f}^{\gamma}(A)+c=m_{f+c}^{\gamma}(A)$ for every $c \in \mathbb{R}$.

(5) $m_{c f}^{\gamma}(A)=c m_{f}^{\gamma}(A)$ for every $c>0$.

(6) $\left|m_{f}^{\gamma}(A)\right| \leq m_{|f|}^{\min \{\gamma, 1-\gamma\}}(A)$.

(7) $m_{f+g}^{\gamma}(A) \leq m_{f}^{\gamma_{1}}(A)+m_{g}^{\gamma_{2}}(A)$ whenever $\gamma_{1}+\gamma_{2}=\gamma$.

(8) For every $p>0$,

$$
m_{|f|}^{\gamma}(A) \leq\left(\gamma^{-1} f_{A}|f|^{p} d \mu\right)^{1 / p}
$$

(9) If $\mathcal{B}$ is a differentiation basis and $f$ is continuous, then for every $x \in X$,

$$
\lim _{\mathcal{B} \ni B \rightarrow x} m_{f}^{\gamma}(B)=u(x) .
$$

Property (9) above asserts that the pointwise value of a continous function can be obtained as a limit of medians over small balls. In this sense medians behave like integral averages of continuous functions.

2.3. Differentiation bases. A collection $\mathcal{B}=\cup_{x \in X} \mathcal{B}(x)$, where $\mathcal{B}(x)$ consist of bounded measurable sets containing $x$, is called a differentiation basis if, for every $x \in \mathbb{R}^{n}$ and $\varepsilon>0$, there exists $B \in \mathcal{B}(x)$ such that $\operatorname{diam}(B)<\varepsilon$. Let $f \in L^{0}(X)$. For a diffentiation basis $\mathcal{B}$, we denote

$$
\begin{gathered}
\left.\limsup _{\mathcal{B} \ni B \rightarrow x} m_{f}^{\gamma}(B)=\limsup _{\varepsilon \rightarrow 0} \sup \gamma(B): B \in \mathcal{B}(x), \operatorname{diam}(B)<\varepsilon\right\}, \\
\liminf _{\mathcal{B} \ni B \rightarrow x} m_{f}^{\gamma}(B)=\lim _{\varepsilon \rightarrow 0} \inf \left\{m_{u}^{\gamma}(B): B \in \mathcal{B}(x), \operatorname{diam}(B)<\varepsilon\right\}
\end{gathered}
$$

and

$$
\lim _{\mathcal{B} \ni B \rightarrow x} m_{f}^{\gamma}(B)=\limsup _{\mathcal{B} \ni B \rightarrow x} m_{f}^{\gamma}(B),
$$

if the limes superior and the limes inferior coincide.

\section{Definition 2.2.}


(1) A differentiation basis $\mathcal{B}$ is a Busemann-Feller basis if each $B \in \mathcal{B}$ is open and $x \in B \in \mathcal{B}$ implies that $B \in \mathcal{B}(x)$.

(2) A differentiation basis $\mathcal{B}$ is a density basis if, for every measurable $A \subset X$,

$$
\lim _{\mathcal{B} \ni B \rightarrow x} \frac{\mu(A \cap B)}{\mu(B)}=\chi_{A}(x)
$$

for almost every $x \in X$.

(3) A differentiation basis $\mathcal{B}$ is homothecy invariant if $B \in \mathcal{B}$ implies that $B^{\prime} \in \mathcal{B}$ for every $B^{\prime}$ homothetic to $B$.

We begin with a version of the Lebesgue density theorem for $\gamma$-medians.

Theorem 2.3. The following claims are equivalent.

(1) $\mathcal{B}$ is a density basis.

(2) For every $f \in L^{0}(X)$, there exists a set $E$ with measure zero such that

$$
\lim _{\mathcal{B} \ni B \rightarrow x} m_{|f-f(x)|}^{\gamma}(B)=0
$$

for every $0<\gamma<1$ and $x \in X \backslash E$.

(3) For every $f \in L^{0}(X)$, there exists a set $E$ with measure zero such that

$$
\lim _{\mathcal{B} \ni B \rightarrow x} m_{f}^{\gamma}(B)=f(x)
$$

for every $0<\gamma<1$ and $x \in X \backslash E$.

Proof. We begin with showing that (1) implies (2). First we prove that for fixed $f \in L^{0}(X)$ and $0<\gamma<1$,

$$
\lim _{\mathcal{B} \ni B \rightarrow x} m_{f}^{\gamma}(B)=f(x)
$$

for almost every $x \in X$. The proof is a slight modification of the proof of [28, Theorem 3.1]. For $k=1,2, \ldots$ and $j \in \mathbb{Z}$, denote

$$
E_{k, j}=\left\{x:(j-1) 2^{-k} \leq f(x)<j 2^{-k}\right\}
$$

and

$$
S_{k}=\sum_{j \in \mathbb{Z}}(j-1) 2^{-k} \chi_{E_{k, j}}
$$

Then $\mu\left(X \backslash \cup_{k, j} E_{k, j}\right)=0$. Since $S_{k} \leq u \leq S_{k}+2^{-k}$ almost everywhere, we have

$$
m_{S_{k}}^{\gamma}(B) \leq m_{f}^{\gamma}(B) \leq m_{S_{k}}^{\gamma}(B)+2^{-k}
$$

for every $B \in \mathcal{B}$. Denote

$$
A_{k, j}=\left\{x \in E_{k, j}: \lim _{\mathcal{B} \ni B \rightarrow x} \frac{\mu\left(E_{k, j} \cap B\right)}{\mu(B)}=1\right\}
$$

and $A=\cup_{k=1}^{\infty} \cup_{j \in \mathbb{Z}} A_{k, j}$. Then, by assumption, $\mu(X \backslash A)=0$. We show that

$$
\lim _{\mathcal{B} \ni B \rightarrow x} m_{f}^{\gamma}(B)=f(x)
$$


for every $x \in A$. Let $x \in A$ and $\varepsilon>0$. Choose $k$ such that $2^{-k+1}<\varepsilon$. Then $x \in A_{k, j}$ for some $j$. Thus, for all $B \in \mathcal{B}$ with $\operatorname{diam}(B)$ small enough,

$$
\frac{\mu\left(E_{k, j} \cap B\right)}{\mu(B)}>\max \{\gamma, 1-\gamma\}
$$

For such $B$, we have

$$
m_{S_{k}}^{\gamma}(B)=(j-1) 2^{-k}
$$

Indeed,

$$
\begin{aligned}
\mu\left(\left\{y \in B: S_{k}(y)>(j-1) 2^{-k}\right\}\right) & \leq \mu\left(B \backslash E_{k, j}\right) \\
& =\mu(B)-\mu\left(B \cap E_{k, j}\right)<\gamma \mu(B)
\end{aligned}
$$

and thus $m_{S_{k}}^{\gamma}(B) \leq(j-1) 2^{-k}$. On the other hand, for every $\delta>0$,

$$
\mu\left(\left\{y \in B: S_{k}(y)>(j-1) 2^{-k}-\delta\right\}\right) \geq \mu\left(B \cap E_{k, j}\right)>\gamma \mu(B)
$$

which implies that $m_{S_{k}}^{\gamma}(B) \geq(j-1) 2^{-k}$. By combining (2.2) and (2.3), we obtain

$$
\left|m_{f}^{\gamma}(B)-u(x)\right| \leq\left|m_{f}^{\gamma}(B)-m_{S_{k}}^{\gamma}(B)\right|+\left|m_{S_{k}}^{\gamma}(B)-f(x)\right| \leq 2^{-k+1}<\varepsilon
$$

for every $B \in \mathcal{B}$ with $\operatorname{diam}(B)$ small enough.

Then assume that $f \in L^{0}(X)$. By what we have shown above, there exists $E \subset X$ with $\mu(E)=0$ such that

$$
\lim _{\mathcal{B} \ni B \rightarrow x} m_{|f-q|}^{\eta}(B)=|f(x)-q|
$$

whenever $\eta \in \mathbb{Q} \cap[0,1], q \in \mathbb{Q}$ and $x \in X \backslash E$. If $0<\gamma<1, x \in X \backslash E$ and $\varepsilon>0$, we choose $q \in \mathbb{Q}$ such that $|f(x)-q|<\varepsilon$ and $\eta \in \mathbb{Q}$ such that $0<\eta \leq \gamma$. This implies

$$
\lim _{\mathcal{B} \ni B \rightarrow x} m_{|f-f(x)|}^{\gamma}(B) \leq \lim _{\mathcal{B} \ni B \rightarrow x} m_{|f-f(x)|}^{\eta}(B)<\lim _{\mathcal{B} \ni B \rightarrow x} m_{|f-q|}^{\eta}(B)+\varepsilon<2 \varepsilon,
$$

which concludes the proof that (1) implies (2).

Then we show that (2) implies (3). Since

$$
\left|f(x)-m_{f}^{\gamma}(B)\right|=\left|m_{(f-f(x))}^{\gamma}(B)\right| \leq m_{|f-f(x)|}^{\min \{\gamma, 1-\gamma\}}(B),
$$

the claim follows immediately.

Finally, we conclude that (3) implies (1). It is easy to see that

$$
m_{\chi_{A}}^{\gamma}(B)=\chi_{(0, \mu(A \cap B) / \mu(B)]}(\gamma)
$$

for every $0<\gamma<1$ and $A, B \subset X$. This completes the proof.

\section{Proof of Theorem 1.1}

Next, we prove that if $\mathcal{B}$ is a homothecy invariant Busemann-Feller density basis on $\mathbb{R}^{n}$, then $\mathcal{M}_{\mathcal{B}}^{\gamma}$ is bounded on $L^{p}\left(\mathbb{R}^{n}\right)$ for $p \geq 0$. The main ingredient of the proof is the following characterization of a density basis ([1, Theorem 1.2]).

Theorem 3.1. Let $\mathcal{B}$ be a homothecy invariant Busemann-Feller basis on $\mathbb{R}^{n}$. Then the following are equivalent.

(1) $\mathcal{B}$ is a density basis. 
(2) For every $0<\gamma<1$, there exists a constant $C=C(\gamma)$ such that

$$
\left|\left\{x \in \mathbb{R}^{n}: \mathcal{M}_{\mathcal{B}} \chi_{A}(x)>\gamma\right\}\right| \leq C|A|
$$

for every bounded measurable set $A \subset \mathbb{R}^{n}$.

Remark 3.2. If (3.1) holds for every bounded measurable set $A \subset \mathbb{R}^{n}$, then it holds for every measurable $A \subset \mathbb{R}^{n}$. Indeed, if $A$ is measurable, then $A_{k}=A \cap B(0, k)$ is bounded and measurable for every $k$ and so

$$
\begin{aligned}
\left|\left\{x \in \mathbb{R}^{n}: \mathcal{M}_{\mathcal{B}} \chi_{A}(x)>\gamma\right\}\right| & =\lim _{k \rightarrow \infty}\left|\left\{x \in \mathbb{R}^{n}: \mathcal{M}_{\mathcal{B}} \chi_{A_{k}}(x)>\gamma\right\}\right| \\
& \leq C \lim _{k \rightarrow \infty}\left|A_{k}\right|=C|A| .
\end{aligned}
$$

We also need the following simple lemma which follows easily from the definitions.

Lemma 3.3. For every $f \in L^{0}\left(\mathbb{R}^{n}\right), x \in \mathbb{R}^{n}, 0<\gamma<1$ and $\lambda>0$, we have

$$
\mathcal{M}_{\mathcal{B}}^{\gamma} f(x)>\lambda \Longleftrightarrow \mathcal{M}_{\mathcal{B}} \chi_{\{|f|>\lambda\}}(x)>\gamma \text {. }
$$

Theorem 3.4. Let $\mathcal{B}$ be a homothecy invariant Busemann-Feller basis on $\mathbb{R}^{n}$. If $\mathcal{B}$ is a density basis then, for every $0<\gamma<1$, there exists a constant $C=C(\gamma)$ such that

$$
\left|\left\{x \in \mathbb{R}^{n}: \mathcal{M}_{\mathcal{B}}^{\gamma} f(x)>\lambda\right\}\right| \leq C\left|\left\{x \in \mathbb{R}^{n}:|f(x)|>\lambda\right\}\right|
$$

for every $f \in L^{0}\left(\mathbb{R}^{n}\right)$ and $\lambda>0$. Consequently, for every $p>0$,

$$
\left\|\mathcal{M}_{\mathcal{B}}^{\gamma} f\right\|_{L^{p}\left(\mathbb{R}^{n}\right)} \leq(C p)^{\frac{1}{p}}\|f\|_{L^{p}\left(\mathbb{R}^{n}\right)}
$$

for every $f \in L^{p}\left(\mathbb{R}^{n}\right)$.

Proof. By Lemma 3.3,

$$
\left|\left\{x \in \mathbb{R}^{n}: \mathcal{M}_{\mathcal{B}}^{\gamma} f(x)>\lambda\right\}\right|=\left|\left\{x \in \mathbb{R}^{n}: \mathcal{M}_{\mathcal{B}} \chi_{\{|f|>\lambda\}}(x)>\gamma\right\}\right|
$$

for every $f \in L^{0}\left(\mathbb{R}^{n}\right), 0<\gamma<1$ and $\lambda>0$. By Theorem 3.1, there exists a constant $C=C(\gamma)$ such that (3.2) holds for every $f \in L^{0}\left(\mathbb{R}^{n}\right)$ and $\lambda>0$. Since

$$
\|f\|_{L^{p}\left(\mathbb{R}^{n}\right)}^{p}=p \int_{0}^{\infty} \lambda^{p-1}\left|\left\{x \in \mathbb{R}^{n}:|f(x)|>\lambda\right\}\right| d \lambda
$$

estimate (3.3) follows from (3.2).

Remark 3.5. Inequality (3.2) implies that $\mathcal{M}_{\mathcal{B}}^{\gamma}$ is also bounded on other rearrangement invariant spaces such as Orlicz and Lorentz spaces.

Next, we prove the implication $(6) \Longrightarrow(5)$ of Theorem 1.1. We need a couple of simple lemmas.

Lemma 3.6. Let $A \subset X$ be a measurable set with $0<\mu(A)<\infty, f \in L^{0}(A)$ and $0<\gamma<1$. Then

$$
\lim _{0<\varepsilon \rightarrow 0} m_{f}^{\gamma-\varepsilon}(A)=m_{f}^{\gamma}(A)
$$


Proof. Let $\lambda>m_{f}^{\gamma}(A)$. Then

$$
\mu(\{x \in A: f(x)>\lambda\})<\gamma \mu(A) .
$$

Thus for $\varepsilon$ small enough,

$$
\mu(\{x \in A: f(x)>\lambda\})<(\gamma-\varepsilon) \mu(A),
$$

which implies that $m_{f}^{\gamma-\varepsilon}(A) \leq \lambda$. This implies that

$$
\limsup _{0<\varepsilon \rightarrow 0} m_{f}^{\gamma-\varepsilon}(A) \leq m_{f}^{\gamma}(A) .
$$

Since $m^{\gamma}(A) \leq m_{f}^{\gamma-\varepsilon}(A)$ for every $0<\varepsilon<\gamma$, the claim follows.

Lemma 3.7. Let $A \subset X$ be a measurable set with $0<\mu(A)<\infty$ and $0<\gamma<1$. If $f_{i} \in L^{0}(A), i=1,2, \ldots$, and $f_{i} \rightarrow f$ in $L^{0}(A)$ as $i \rightarrow \infty$, then

$$
\lim _{i \rightarrow \infty} m_{f_{i}}^{\gamma}(A)=m_{f}^{\gamma}(A) \text {. }
$$

Proof. Let $\lambda>m_{u}^{\gamma}(A)$. Then

$$
\mu(\{x \in A: f(x)>\lambda\})<\gamma \mu(A) .
$$

For $\varepsilon>0$, we have

$$
\begin{aligned}
\left.\mu\left\{x \in A: f_{i}(x)>\lambda+\varepsilon\right\}\right) & \leq \mu(\{x \in A: f(x)>\lambda\}) \\
& +\mu\left(\left\{x \in A:\left|f_{i}(x)-f(x)\right|>\varepsilon\right\}\right)<\gamma \mu(A)
\end{aligned}
$$

for $i$ large enough, which implies that

$$
\limsup _{i \rightarrow \infty} m_{f_{i}}^{\gamma}(A) \leq \lambda+\epsilon
$$

Thus

$$
\limsup _{i \rightarrow \infty} m_{f_{i}}^{\gamma}(A) \leq m_{f}^{\gamma}(A)
$$

Then we show that

$$
m_{f}^{\gamma}(A) \leq \liminf _{i \rightarrow \infty} m_{f_{i}}^{\gamma}(A)
$$

Let

$$
\lambda>\liminf _{i \rightarrow \infty} m_{f_{i}}^{\gamma}(A) .
$$

Then there are arbitrarily large $i$ such that

$$
\mu\left(\left\{x \in A: f_{i}(x)>\lambda\right\}\right)<\gamma \mu(A),
$$

which implies that, for $\varepsilon, \delta>0$,

$$
\begin{aligned}
\mu(\{x \in A: f(x)>\lambda+\varepsilon\}) & \leq \liminf _{i \rightarrow \infty} \mu\left(\left\{x \in A: f_{i}(x)>\lambda\right\}\right) \\
& +\lim _{i \rightarrow \infty} \mu\left(\left\{x \in A:\left|f(x)-f_{i}(x)\right|>\varepsilon\right\}\right) \\
& \leq \gamma \mu(A)<(\gamma-\delta) \mu(A) .
\end{aligned}
$$

Thus, $m_{f}^{\gamma-\delta}(A) \leq \lambda+\varepsilon$. By Lemma 3.6, $m_{f}^{\gamma}(A) \leq \lambda+\varepsilon$. The claim follows by passing $\varepsilon \rightarrow 0$ and $\lambda \rightarrow \liminf _{i \rightarrow \infty} m_{f_{i}}^{\gamma}(A)$. 
Lemma 3.8. Let $A_{i} \subset X, i=1,2, \ldots$, be measurable sets such that $A_{i} \subset A_{i+1}$ for every $i$ and $A=\cup_{i=1}^{\infty} A_{i}$ is of finite measure. Then, for every $0<\gamma<1$ and $f \in L^{0}(X)$, we have

$$
\lim _{i \rightarrow \infty} m_{f}^{\gamma}\left(A_{i}\right)=m_{f}^{\gamma}(A) .
$$

Proof. It suffices to show that

$$
\liminf _{i \rightarrow \infty} m_{f}^{\gamma}\left(A_{i}\right) \geq m_{f}^{\gamma}(A) .
$$

Let $\varepsilon, \delta>0$. By definition,

$$
\mu\left(\left\{x \in A: f(x)>m_{u}^{\gamma-\delta}(A)-\varepsilon\right\}\right) \geq(\gamma-\delta) \mu(A) .
$$

It follows that there is $i_{0}=i_{0}(\delta)$ such that

$$
\mu\left(\left\{x \in A_{i}: f(x)>m_{f}^{\gamma-\delta}(A)-\varepsilon\right\}\right)>\gamma \mu\left(A_{i}\right)
$$

when $i \geq i_{0}$. Thus

$$
\liminf _{i \rightarrow \infty} m_{f}^{\gamma}\left(A_{i}\right) \geq m_{f}^{\gamma-\delta}(A)-\varepsilon .
$$

The claim follows by letting $\delta, \varepsilon \rightarrow 0$.

Lemma 3.9. Let $X$ be separable and let $\mathcal{B}$ be a Busemann-Feller basis on $X$. Then there exists a countable Busemann-Feller basis $\mathcal{B}^{\prime}$ such that

$$
\mathcal{M}_{\mathcal{B}}^{\gamma} f(x) \leq \mathcal{M}_{\mathcal{B}^{\prime}}^{\gamma} f(x) \leq \mathcal{M}_{\mathcal{B}}^{\gamma / 2} f(x)
$$

for every $0<\gamma<1, f \in L^{0}(X)$ and $x \in X$.

Proof. Fix a countable dense set $A \subset X$. Then

$$
\mathcal{C}=\left\{\cup_{i=1}^{k} B\left(x_{i}, r_{i}\right): k \in \mathbb{N}, x_{i} \in A, r_{i} \in \mathbb{Q}\right\}
$$

is countable. Since $B \in \mathcal{B}$ is open,

$$
B=\cup\{B(x, r): x \in B \cap A, r \in \mathbb{Q}, B(x, r) \subset B\} .
$$

Hence, for every $B \in \mathcal{B}$, there exists a nondecreasing sequence $\left(C_{i}\right), C_{i} \in \mathcal{C}$, such that $\cup_{i=1}^{\infty} C_{i}=B$. It follows that $\lim _{i \rightarrow \infty} \mu\left(C_{i}\right)=\mu(B)$ and, by Lemma 3.8,

$$
\lim _{i \rightarrow \infty} m_{|f|}^{\gamma}\left(C_{i}\right)=m_{|f|}^{\gamma}(B)
$$

for every $0<\gamma<1$ and $f \in L^{0}(X)$. Also, if $x \in B$, then $x \in C_{i}$ for large enough $i$. Define

$$
\mathcal{B}^{\prime}(x)=\left\{C \in \mathcal{C}: x \in C, C \subset B \text { for some } B \in \mathcal{B} \text { and } \mu(C) \geq \frac{1}{2} \mu(B)\right\} .
$$

Then $\mathcal{B}^{\prime}=\cup_{x \in X} \mathcal{B}^{\prime}(x)$ is clearly a Busemann-Feller basis. By (3.5),

$$
\mathcal{M}_{\mathcal{B}}^{\gamma} f(x) \leq \mathcal{M}_{\mathcal{B}^{\prime}}^{\gamma} f(x)
$$

for every $0<\gamma<1, f \in L^{0}(X)$ and $x \in X$. If $C \in \mathcal{B}^{\prime}$, there exists $B \in \mathcal{B}$ such that $C \subset B$ and $\mu(B) \leq 2 \mu(C)$. By Lemma 2.1(3), $m_{|f|}^{\gamma}(C) \leq m_{|f|}^{\gamma / 2}(B)$ for every $0<\gamma<1$ and $f \in L^{0}(X)$. It follows that

$$
\mathcal{M}_{\mathcal{B}^{\prime}}^{\gamma} f(x) \leq \mathcal{M}_{\mathcal{B}}^{\gamma / 2} f(x)
$$

for every $0<\gamma<1, f \in L^{0}(X)$ and $x \in X$. 
Theorem 3.10. Assume that $X$ is separable and let $\mathcal{B}$ be a Busemann-Feller basis on $X$. Let $0<\gamma<1$ and $0<p<\infty$. Assume that, for every $f \in L^{p}(X)$, $\mathcal{M}_{\mathcal{B}}^{\gamma / 4} f(x)<\infty$ for almost every $x \in X$. Then, for every $\lambda>0$,

$$
\mu\left(\left\{x \in X: \mathcal{M}_{\mathcal{B}}^{\gamma} f_{i}(x)>\lambda\right\}\right) \rightarrow 0
$$

as $\left\|f_{i}\right\|_{L^{p}(X)} \rightarrow 0$.

Proof. The set $L^{0}(X)$ of measurable almost everywhere finite functions equipped with a functional

$$
\|f\|_{L^{0}(X)}=\inf _{\lambda>0}\{\lambda+\mu(\{x \in X:|u(x)|>\lambda\})\}
$$

is a quasi-normed space in the sense of Yosida [32, p. 30]. In particular,

$$
\|f+g\|_{L^{0}(X)} \leq\|g\|_{L^{0}(X)}+\|g\|_{L^{0}(X)}
$$

for every $f, g \in L^{0}(X)$ and

$$
\left\|a_{m} f\right\|_{L^{0}(X)} \rightarrow 0 \text { whenever } f \in L^{0}(X) \text { and } a_{m} \rightarrow 0 .
$$

It is easy to see that

$$
\lim _{i \rightarrow \infty} \mu\left(\left\{x \in X:\left|f_{i}(x)-f(x)\right|>\lambda\right\}\right)=0,
$$

for every $\lambda>0$, if and only if $\lim _{i \rightarrow \infty}\left\|f_{i}-f\right\|_{L^{0}(X)}=0$.

By Lemma 3.9, there exists a countable Busemann-Feller basis $\mathcal{B}^{\prime}=\left\{B_{j}: j=\right.$ $1,2, \ldots\}$ such that

$$
\mathcal{M}_{\mathcal{B}}^{\gamma} f(x) \leq \mathcal{M}_{\mathcal{B}^{\prime}}^{\gamma} f(x)
$$

and

$$
\mathcal{M}_{\mathcal{B}^{\prime}}^{\gamma / 2} f(x) \leq \mathcal{M}_{\mathcal{B}}^{\gamma / 4} f(x)
$$

for every $f \in L^{0}(X)$ and $x \in X$. For $k=1,2, \ldots$, denote

$$
\mathcal{M}_{k}^{\gamma / 2} f(x)=\max \left\{m_{|f|}^{\gamma / 2}\left(B_{j}\right): B_{j} \ni x, 1 \leq j \leq k\right\} .
$$

By Lemma 3.7, operators $\mathcal{M}_{k}^{\gamma / 2}$ are continuous from $L^{p}(X), p \geq 0$, to $L^{0}(X)$. Let $\varepsilon>0$. For $m \in \mathbb{N}$, denote

$$
F_{m}=\left\{f \in L^{p}(X): \sup _{k}\left\|m^{-1} M_{k}^{\gamma / 2} f\right\|_{L^{0}(X)} \leq \varepsilon\right\} .
$$

By continuity, sets $F_{m}$ are closed. Since, by assumption and (3.9), $\mathcal{M}_{\mathcal{B}^{\prime}}^{\gamma / 2} f \in L^{0}(X)$, (3.7) implies that

$$
\sup _{k}\left\|m^{-1} M_{k}^{\gamma / 2} f\right\|_{L^{0}(X)} \leq\left\|m^{-1} M_{\mathcal{B}^{\prime}}^{\gamma / 2} f\right\|_{L^{0}(X)} \rightarrow 0
$$

as $m \rightarrow \infty$. It follows that $L^{p}(X)=\cup_{m=1}^{\infty} F_{m}$. By the Baire-Hausdorff theorem ([32, p.11]), one of the sets $F_{m}$ must have non-empty interior. Thus, there is $m_{0} \in \mathbb{N}$, $f_{0} \in F_{m_{0}}$ and $\delta>0$ such that $\|f\|_{L^{p}(X)}<\delta$ implies that $f_{0}+f \in F_{m_{0}}$. Hence, if $\|f\|_{L^{p}(X)}<\delta$, we have

$$
\left\|\mathcal{M}_{k}^{\gamma} m_{0}^{-1} f\right\|_{L^{0}(X)} \leq\left\|m_{0}^{-1} \mathcal{M}_{k}^{\gamma / 2}\left(f_{0}+f\right)\right\|_{L^{0}(X)}+\left\|m_{0}^{-1} \mathcal{M}_{k}^{\gamma / 2} f_{0}\right\|_{L^{0}(X)} \leq 2 \varepsilon
$$


for every $k \in \mathbb{N}$. It follows that $\sup _{k}\left\|\mathcal{M}_{k}^{\gamma} f_{i}\right\|_{L^{0}(X)} \rightarrow 0$ as $\left\|f_{i}\right\|_{L^{p}(X)} \rightarrow 0$. This and (3.8) imply that, for every $\lambda>0$,

$$
\begin{aligned}
\mu\left(\left\{x \in X: \mathcal{M}_{\mathcal{B}}^{\gamma} f_{i}(x)>\lambda\right\}\right) & \leq \mu\left(\left\{x \in X: \mathcal{M}_{\mathcal{B}^{\prime}}^{\gamma} f_{i}(x)>\lambda\right\}\right) \\
& =\sup _{k} \mu\left(\left\{x \in X: \mathcal{M}_{k}^{\gamma} f_{i}(x)>\lambda\right\}\right) \rightarrow 0
\end{aligned}
$$

as $\left\|f_{i}\right\|_{L^{p}(X)} \rightarrow 0$.

To prove implication $(5) \Longrightarrow(1)$ of Theorem 1.1, we employ yet another characterization of a density basis, see [1, Theorem 1.1].

Theorem 3.11. Let $\mathcal{B}$ be a Busemann-Feller basis. Then the following are equivalent.

(1) $\mathcal{B}$ is a density basis.

(2) For every $0<\gamma<1$, for every nonincreasing sequence $\left(r_{k}\right)$ such that $r_{k} \rightarrow 0$ as $k \rightarrow \infty$, and for every nonincreasing sequence $\left(A_{k}\right)$ of bounded measurable sets such that $\left|A_{k}\right| \rightarrow 0$ as $k \rightarrow \infty$, we have

$$
\left|\left\{x \in \mathbb{R}^{n}: \mathcal{M}_{\mathcal{B}, r_{k}} \chi_{A_{k}}(x)>\gamma\right\}\right| \rightarrow 0
$$

as $k \rightarrow \infty$.

Proof of implication (5) $\Longrightarrow$ (1) of Theorem 1.1. By Theorem 3.11, it suffices to show that

$$
\left|\left\{x \in \mathbb{R}^{n}: \mathcal{M}_{\mathcal{B}} \chi_{A_{k}}(x)>\gamma\right\}\right| \rightarrow 0
$$

as $k \rightarrow \infty$, whenever $0<\gamma<1$ and $\left(A_{k}\right)$ is a sequence of bounded measurable sets such that $\left|A_{k}\right| \rightarrow 0$ as $k \rightarrow \infty$. Let $\left(A_{k}\right)$ be such a sequence. Then, $\left\|\chi_{A_{k}}\right\|_{L^{p}(X)} \rightarrow 0$ as $k \rightarrow \infty$. By applying Lemma 3.3 with $f=\chi_{A_{k}}$ and $\lambda=1 / 2$, we have

$$
\left|\left\{x \in \mathbb{R}^{n}: \mathcal{M}_{\mathcal{B}} \chi_{A_{k}}(x)>\gamma\right\}\right|=\left|\left\{x \in \mathbb{R}^{n}: \mathcal{M}_{\mathcal{B}}^{\gamma} \chi_{A_{k}}(x)>1 / 2\right\}\right|,
$$

which, by assumption, tends to zero as $k \rightarrow \infty$.

\section{HajŁasz spaces, HajŁasz-Besov and HajŁasz-Triebel-Lizorkin SPACES}

Among several definitions for Besov and Triebel-Lizorkin spaces in metric measure spaces, we use the one introduced in [23]. This definition is motivated by the Hajłasz-Sobolev spaces $M^{s, p}(X)$, defined for $s=1, p \geq 1$ in [6] and for fractional scales in [32]. We begin with the definition of a gradient on metric measure spaces.

Definition 4.1. Let $0<s<\infty$. A measurable function $g: X \rightarrow[0, \infty]$ is an s-gradient of a function $u \in L^{0}(X)$ if there exists a set $E \subset X$ with $\mu(E)=0$ such that for all $x, y \in X \backslash E$,

$$
|u(x)-u(y)| \leq d(x, y)^{s}(g(x)+g(y)) .
$$

The collection of all $s$-gradients of $u$ is denoted by $\mathcal{D}^{s}(u)$.

Then we define Sobolev spaces on metric measure spaces. 
Definition 4.2. Let $0<p \leq \infty$. The homogeneous Hajtasz space $\dot{M}^{s, p}(X)$ consists of measurable functions $u$ for which

$$
\|u\|_{\dot{M}^{s, p}(X)}=\inf _{g \in \mathcal{D}^{s}(u)}\|g\|_{L^{p}(X)}
$$

is finite. The Hajtasz space $M^{s, p}(X)$ is $\dot{M}^{s, p}(X) \cap L^{p}(X)$ equipped with the norm

$$
\|u\|_{M^{s, p}(X)}=\|u\|_{L^{p}(X)}+\|u\|_{\dot{M}^{s, p}(X)} .
$$

Recall that for $p>1, M^{1, p}\left(\mathbb{R}^{n}\right)=W^{1, p}\left(\mathbb{R}^{n}\right)$, see $[6]$, whereas for $n /(n+1)<p \leq 1$, $M^{1, p}\left(\mathbb{R}^{n}\right)$ coincides with the Hardy-Sobolev space $H^{1, p}\left(\mathbb{R}^{n}\right)$ by $[22$, Theorem 1$]$.

For the definition of Hajłasz-Triebel-Lizorkin and Hajłasz-Besov spaces, we need a concept of a fractional gradient, which consists of a sequence of gradient functions.

Definition 4.3. Let $0<s<\infty$. A sequence of nonnegative measurable functions $\left(g_{k}\right)_{k \in \mathbb{Z}}$ is a fractional s-gradient of a function $u \in L^{0}(X)$, if there exists a set $E \subset X$ with $\mu(E)=0$ such that

$$
|u(x)-u(y)| \leq d(x, y)^{s}\left(g_{k}(x)+g_{k}(y)\right)
$$

for all $k \in \mathbb{Z}$ and all $x, y \in X \backslash E$ satisfying $2^{-k-1} \leq d(x, y)<2^{-k}$. The collection of all fractional $s$-gradients of $u$ is denoted by $\mathbb{D}^{s}(u)$.

For $0<p, q \leq \infty$ and a sequence $\left(f_{k}\right)_{k \in \mathbb{Z}}$ of measurable functions, we define

$$
\left\|\left(f_{k}\right)_{k \in \mathbb{Z}}\right\|_{L^{p}\left(X, l^{q}\right)}=\|\|\left(f_{k}\right)_{k \in \mathbb{Z}}\left\|_{l^{q}}\right\|_{L^{p}(X)}
$$

and

$$
\left\|\left(f_{k}\right)_{k \in \mathbb{Z}}\right\|_{l^{q}\left(L^{p}(X)\right)}=\left\|\left(\left\|f_{k}\right\|_{L^{p}(X)}\right)_{k \in \mathbb{Z}}\right\|_{l^{q}},
$$

where

$$
\left\|\left(f_{k}\right)_{k \in \mathbb{Z}}\right\|_{l^{q}}= \begin{cases}\left(\sum_{k \in \mathbb{Z}}\left|f_{k}\right|^{q}\right)^{1 / q}, & \text { if } 0<q<\infty \\ \sup _{k \in \mathbb{Z}}\left|f_{k}\right|, & \text { if } q=\infty .\end{cases}
$$

Next we recall the definition of Hajłasz-Triebel-Lizorkin and Hajłasz-Besov spaces on metric measure spaces.

Definition 4.4. Let $0<s<\infty$ and $0<p, q \leq \infty$.

(1) The homogeneous Hajtasz-Triebel-Lizorkin space $\dot{M}_{p, q}^{s}(X)$ consists of functions $u \in L^{0}(X)$, for which the (semi)norm

$$
\|u\|_{\dot{M}_{p, q}^{s}(X)}=\inf _{\left(g_{k}\right) \in \mathbb{D}^{s}(u)}\left\|\left(g_{k}\right)\right\|_{L^{p}\left(X, l^{q}\right)}
$$

is finite. The Hajtasz-Triebel-Lizorkin space $M_{p, q}^{s}(X)$ is $\dot{M}_{p, q}^{s}(X) \cap L^{p}(X)$ equipped with the norm

$$
\|u\|_{M_{p, q}^{s}(X)}=\|u\|_{L^{p}(X)}+\|u\|_{\dot{M}_{p, q}^{s}(X)} .
$$

Notice that $M_{p, \infty}^{s}(X)=M^{s, p}(X)$, see [23, Prop. 2.1]. 
(2) Similarly, the homogeneous Hajtasz-Besov space $\dot{N}_{p, q}^{s}(X)$ consists of functions $u \in L^{0}(X)$, for which

$$
\|u\|_{\dot{N}_{s, q}^{s}(X)}=\inf _{\left(g_{k}\right) \in \mathbb{D}^{s}(u)}\left\|\left(g_{k}\right)\right\|_{l^{q}\left(L^{p}(X)\right)}
$$

is finite, and the Hajtasz-Besov space $N_{p, q}^{s}(X)$ is $\dot{N}_{p, q}^{s}(X) \cap L^{p}(X)$ equipped with the norm

$$
\|u\|_{N_{p, q}^{s}(X)}=\|u\|_{L^{p}(X)}+\|u\|_{\dot{N}_{p, q}^{s}(X)} .
$$

For $0<s<1,0<p, q \leq \infty$, the spaces $N_{p, q}^{s}\left(\mathbb{R}^{n}\right)$ and $M_{p, q}^{s}\left(\mathbb{R}^{n}\right)$ coincide with the classical Besov and Triebel-Lizorkin spaces defined via differences, see [5].

When $0<p<1$ or $0<q<1$, the (semi)norms defined above are actually quasi(semi)norms, but for simplicity we call them, as well as other quasi-seminorms in this paper, just norms.

Definition 4.5. Let $0<s<\infty, 0<p, q \leq \infty$ and $\mathcal{F} \in\left\{N_{p, q}^{s}(X), M_{p, q}^{s}(X)\right\}$. The $\mathcal{F}$-capacity of a set $E \subset X$ is

$$
C_{\mathcal{F}}(E)=\inf \left\{\|u\|_{\mathcal{F}}^{p}: u \in \mathcal{A}_{\mathcal{F}}(E)\right\}
$$

where

$$
\mathcal{A}_{\mathcal{F}}(E)=\{u \in \mathcal{F}: u \geq 1 \text { on a neighbourhood of } E\}
$$

is a set of admissible functions for the capacity. We say that a property holds $\mathcal{F}$-quasieverywhere if it holds outside a set of $\mathcal{F}$-capacity zero.

Remark 4.6. It is easy to see that

$$
C_{\mathcal{F}}(E)=\inf \left\{\|u\|_{\mathcal{F}}^{p}: u \in \mathcal{A}_{\mathcal{F}}^{\prime}(E)\right\},
$$

where $\mathcal{A}_{\mathcal{F}}^{\prime}(E)=\left\{u \in \mathcal{A}_{\mathcal{F}}(E): 0 \leq u \leq 1\right\}$.

Remark 4.7. The $\mathcal{F}$-capacity is an outer capacity, which means that

$$
C_{\mathcal{F}}(E)=\inf \left\{C_{\mathcal{F}}(U): U \supset E, U \text { is open }\right\} .
$$

A function $u: X \rightarrow[-\infty, \infty]$ is $\mathcal{F}$-quasicontinuous if for every $\varepsilon>0$, there exists a set $E \subset X$ such that $C_{\mathcal{F}}(E)<\varepsilon$ and the restriction of $u$ to $X \backslash E$ is continuous. The following lemma follows from [9, Theorem 1.2].

Lemma 4.8. For every $u \in \mathcal{F}$, there exists an $\mathcal{F}$-quasicontinuous $u^{*}$ such that $u(x)=u^{*}(x)$ for almost every $x \in X$.

The $\mathcal{F}$-quasicontinuous representative is unique in the sense that if two $\mathcal{F}$-quasicontinuous functions coincide almost everywhere, then they actually coincide $\mathcal{F}$-quasieverywhere, see [19]. The following lemma gives a useful characterization of the capacity in terms of quasicontinuous functions. The proof of the lemma is essentially same as the proof of [20, Theorem 3.4]. For $E \subset X$, denote

$\mathcal{Q A}_{\mathcal{F}}(E)=\{u \in \mathcal{F}: u$ is $\mathcal{F}$-quasicontinuous and $u \geq 1 \mathcal{F}$-quasieverywhere in $E\}$ and

$$
\widetilde{C}_{\mathcal{F}}(E)=\inf _{u \in \mathcal{Q}_{\mathcal{F}}(E)}\|u\|_{\mathcal{F}}^{p} .
$$


Lemma 4.9. Let $0<s \leq 1,0<p<\infty, 0<q \leq \infty$ and $\mathcal{F} \in\left\{M_{p, q}^{s}(X), N_{p, q}^{s}(X)\right\}$. Then there exists a constant $C$ such that

$$
\widetilde{C}_{\mathcal{F}}(E) \leq C_{\mathcal{F}}(E) \leq C \widetilde{C}_{\mathcal{F}}(E)
$$

for every $E \subset X$.

Proof. To prove the first inequality, let $u \in \mathcal{A}_{\mathcal{F}}(E)$ and let $u^{*}$ be a quasicontinuous representative of $u$. Since $u \geq 1$ in some open set $U$ containing $E$ and $u^{*}=u$ almost everywhere, it follows that $\min \left\{0, u^{*}-1\right\}=0$ almost everywhere in $U$. Since $\min \left\{0, u^{*}-1\right\}$ is quasicontinuous, the equality actually holds quasieverywhere in $U$. Hence $u^{*} \geq 1$ quasieverywhere in $U$, which implies that $u^{*} \in \mathcal{Q} \mathcal{A}_{\mathcal{F}}(E)$.

For the second inequality, let $v \in \mathcal{Q}_{\mathcal{F}}(E)$. By truncation, we may assume that $0 \leq u \leq 1$. Fix $0<\varepsilon<1$, and choose an open set $V$ with $C_{\mathcal{F}}(V)<\varepsilon$ so that $v=1$ on $E \backslash V$ and that $v$ is continuous in $X \backslash V$. By continuity, there is an open set $U \subset X$ such that

$$
\{x \in X: v(x)>1-\varepsilon\} \backslash V=U \backslash V .
$$

Clearly, $E \backslash V \subset U \backslash V$. Choose $u \in \mathcal{A}_{\mathcal{F}}(V)$ such that $\|u\|_{\mathcal{F}}<\varepsilon$ and that $0 \leq u \leq 1$. Define $w=v /(1-\varepsilon)+u$. Then $w \geq 1$ on $(U \backslash V) \cup V=U \cup V$ which is an open neighbourhood of $E$. Hence $w \in \mathcal{A}_{\mathcal{F}}(E)$ and so

$$
C_{\mathcal{F}}(E)^{1 / p} \leq\|w\|_{\mathcal{F}} \leq C\left(\frac{1}{1-\varepsilon}\|v\|_{\mathcal{F}}+\|u\|_{\mathcal{F}}\right) \leq C\left(\frac{1}{1-\varepsilon}\|v\|_{\mathcal{F}}+\varepsilon\right) .
$$

Since $\varepsilon>0$ and $v \in \mathcal{Q}_{\mathcal{F}}(E)$ are arbitrary, the desired inequality $C_{\mathcal{F}}(E) \leq C \widetilde{C}_{\mathcal{F}}(E)$ follows.

The $\mathcal{F}$-capacity is not generally subadditive but, for most purposes, the following result is sufficient, see [9, Lemma 6.4].

Lemma 4.10. Let $0<s<\infty, 0<p<\infty, 0<q \leq \infty$ and let $\mathcal{F} \in\left\{N_{p, q}^{s}(X), M_{p, q}^{s}(X)\right\}$. Then there are constants $C \geq 1$ and $0<r \leq 1$ such that

$$
C_{\mathcal{F}}\left(\bigcup_{i \in \mathbb{N}} E_{i}\right)^{r} \leq C \sum_{i \in \mathbb{N}} C_{\mathcal{F}}\left(E_{i}\right)^{r}
$$

for all sets $E_{i} \subset X, i \in \mathbb{N}$. In fact, (4.3) holds with $r=\min \{1, q / p\}$.

The following theorem is the main result of this section. Its proof is a modification of the proof of [7, Theorem 2.11].

Theorem 4.11. Let $\mathcal{B}$ be a differentiation basis on a doubling metric measure space $X$. Let $\mathcal{F} \in\left\{N_{p, q}^{s}(X), M_{p, q}^{s}(X)\right\}$, where $0<s<1,0<p, q<\infty$, or $\mathcal{F}=$ $M_{p, \infty}^{s}(X)=M^{s, p}(X)$, where $0<s \leq 1$ and $0<p<\infty$. Then the following claims are equivalent.

(1) For every quasicontinuous $u \in \mathcal{F}$, there exists a set $E$ with $C_{\mathcal{F}}(E)=0$ such that

$$
\lim _{\mathcal{B} \ni B \rightarrow x} m_{u}^{\gamma}(B)=u(x)
$$

for every $0<\gamma<1$ and $x \in X \backslash E$. 
(2) For every $0<\gamma<1$, there exists a constant $C$ such that

$$
C_{\mathcal{F}}\left(\left\{x \in X: \limsup _{\mathcal{B} \ni B \rightarrow x} m_{|u|}^{\gamma}(B)>\lambda\right\}\right) \leq C \lambda^{-p}\|u\|_{\mathcal{F}}^{p}
$$

for every $\lambda>0$ and $u \in \mathcal{F}$.

(3) For each $0<\gamma<1, \lambda>0$ and for each sequence $\left(u_{k}\right)$ such that $\left\|u_{k}\right\|_{\mathcal{F}} \rightarrow 0$ as $k \rightarrow \infty$, we have

$$
C_{\mathcal{F}}\left(\left\{x \in X: \limsup _{\mathcal{B} \ni B \rightarrow x} m_{\left|u_{k}\right|}^{\gamma}(B)>\lambda\right\}\right) \rightarrow 0
$$

as $k \rightarrow \infty$.

Remark 4.12. If $X$ and $\mathcal{F}$ are as above and $\mathcal{B}=\{B(x, r): x \in X, r>0\}$, then by [9, Theorem 7.7], for every $0<\gamma<1$, there exists a constant $C$ such that

$$
C_{\mathcal{F}}\left(\left\{x \in X: \mathcal{M}_{\mathcal{B}}^{\gamma} u(x)>\lambda\right\}\right) \leq C \lambda^{-p}\|u\|_{\mathcal{F}}^{p}
$$

for every $\lambda>0$ and $u \in \mathcal{F}$. It would be interesting to find out under what assumptions on $X, \mathcal{B}$ and $\mathcal{F}$ this type of estimate is equivalent to conditions (1)-(3) of Theorem 4.11.

For the proof of Theorem 4.11, we need the following lemma.

Lemma 4.13. Let $\mathcal{B}$ be a differentiation basis on a doubling metric measure space $X$. Let $\mathcal{F} \in\left\{N_{p, q}^{s}(X), M_{p, q}^{s}(X)\right\}$, where $0<s<1,0<p, q<\infty$, or $\mathcal{F}=M_{p, \infty}^{s}(X)=$ $M^{s, p}(X)$, where $0<s \leq 1$ and $0<p<\infty$. Let $0<\eta<\gamma<1$. Suppose that, for every $\lambda>0$,

$$
C_{\mathcal{F}}\left(\left\{x \in X: \limsup _{\mathcal{B} \ni B \rightarrow x} m_{\left|u_{k}\right|}^{\eta}(B)>\lambda\right\}\right) \rightarrow 0
$$

as $\left\|u_{k}\right\|_{\mathcal{F}} \rightarrow 0$. Then, for every quasicontinuous $u \in \mathcal{F}$, there exists a set $E$ with $C_{\mathcal{F}}(E)=0$ such that

$$
\lim _{\mathcal{B} \ni B \rightarrow x} m_{|u-u(x)|}^{\gamma}(B)=0
$$

for every $x \in X \backslash E$.

Proof. By [9, Theorem 1.1], if $0<q<\infty$, and by [29, Proposition 4.5], if $q=\infty$, continuous functions are dense in $\mathcal{F}$. Let $u \in \mathcal{F}$ be quasicontinuous and let $v_{k} \in \mathcal{F}$, $k=1,2, \ldots$, be continuous such that $\left\|u-v_{k}\right\|_{\mathcal{F}} \rightarrow 0$ as $k \rightarrow \infty$. Denote $w_{k}=u-v_{k}$. By Lemma 2.1,

$$
\begin{aligned}
\limsup _{\mathcal{B} \ni B \rightarrow x} m_{|u-u(x)|}^{\gamma}(B) & \leq \limsup _{\mathcal{B} \ni B \rightarrow x} m_{\left|v_{k}-v_{k}(x)\right|}^{\gamma-\eta}(B)+\limsup _{\mathcal{B} \ni B \rightarrow x} m_{\left|w_{k}-w_{k}(x)\right|}^{\eta}(B) \\
& \leq \limsup _{\mathcal{B} \ni B \rightarrow x} m_{\left|w_{k}\right|}^{\eta}(B)+\left|w_{k}(x)\right| .
\end{aligned}
$$

Hence, by Lemma 4.10,

$$
\begin{aligned}
& C_{\mathcal{F}}\left(\left\{x \in X: \limsup _{\mathcal{B} \ni B \rightarrow x} m_{|u-u(x)|}^{\gamma}(B)>\lambda\right\}\right)^{r} \\
& \leq C\left(C_{\mathcal{F}}\left(\left\{x \in X: \limsup _{\mathcal{B} \ni B \rightarrow x} m_{\left|w_{k}\right|}^{\eta}(B)>\lambda / 2\right\}\right)^{r}+C_{\mathcal{F}}\left(\left\{x \in X:\left|w_{k}(x)\right|>\lambda / 2\right\}\right)^{r}\right) .
\end{aligned}
$$

By assumption,

$$
C_{\mathcal{F}}\left(\left\{x \in X: \limsup _{\mathcal{B} \ni B \rightarrow x} m_{\left|w_{k}\right|}^{\eta}(B)>\lambda / 2\right\}\right) \rightarrow 0
$$


as $k \rightarrow \infty$. Since $\left|w_{k}\right|$ is quasicontinuous, Lemma 4.9 gives

$$
\begin{aligned}
C_{\mathcal{F}}\left(\left\{x \in X:\left|w_{k}(x)\right|>\lambda / 2\right\}\right) & \leq C \widetilde{C}_{\mathcal{F}}\left(\left\{x \in X:\left|w_{k}(x)\right|>\lambda / 2\right\}\right) \\
& \leq C 2^{p} \lambda^{-p}\left\|w_{k}\right\|_{\mathcal{F}}^{p} \rightarrow 0
\end{aligned}
$$

as $k \rightarrow \infty$. It follows that

$$
C_{\mathcal{F}}\left(\left\{x \in X: \limsup _{\mathcal{B} \ni B \rightarrow x} m_{|u-u(x)|}^{\gamma}(B)>\lambda\right\}\right)=0
$$

for every $\lambda>0$. Hence, by Lemma 4.10 ,

$$
C_{\mathcal{F}}\left(\left\{x \in X: \limsup _{\mathcal{B} \ni B \rightarrow x} m_{|u-u(x)|}^{\gamma}(B)>0\right\}\right)=0 .
$$

Proof of Theorem 4.11. We show that (1) implies (2). Let $0<\gamma<1$ and $u \in \mathcal{F}$. Then $|u| \in \mathcal{F}$ and $\|\mid u\|_{\mathcal{F}} \leq\|u\|_{\mathcal{F}}$. Let $|u|^{*}$ be a quasicontinuous representative of $|u|$ and let $\lambda>0$. By (1) and Lemma 4.9, we have

$$
\begin{aligned}
C_{\mathcal{F}}\left(\left\{x \in X: \limsup _{\mathcal{B} \ni B \rightarrow x} m_{|u|}^{\gamma}(B)>\lambda\right\}\right) & =C_{\mathcal{F}}\left(\left\{x \in X: \limsup _{\mathcal{B} \ni B \rightarrow x} m_{|u|^{*}}^{\gamma}(B)>\lambda\right\}\right) \\
& =C_{\mathcal{F}}\left(\left\{x \in X:|u|^{*}(x)>\lambda\right\}\right) \\
& \leq C \widetilde{C}_{\mathcal{F}}\left(\left\{x \in X:|u|^{*}(x)>\lambda\right\}\right) \\
& \leq C \lambda^{-p}\left\||u|^{*}\right\|_{\mathcal{F}}^{p} \\
& \leq C \lambda^{-p}\|u\|_{\mathcal{F}}^{p} .
\end{aligned}
$$

It is clear that $(2) \Longrightarrow(3)$.

Then we show that (3) implies (1). Let $u \in \mathcal{F}$ be quasicontinuous. By Lemma 4.13 , for every $k=2,3, \ldots$, there exists $E_{k}$ such that $C_{\mathcal{F}}\left(E_{k}\right)=0$ and

$$
\lim _{\mathcal{B} \ni B \rightarrow x} m_{|u-u(x)|}^{1 / k}(B)=0
$$

for every $x \in X \backslash E_{k}$. It follows that

$$
\lim _{\mathcal{B} \ni B \rightarrow x} m_{|u-u(x)|}^{\gamma}(B)=0
$$

for every $0<\gamma<1$ and $x \in X \backslash E$, where $E=\cup_{k \geq 2} E_{k}$ is of $\mathcal{F}$-capacity zero by Lemma 4.10. By Lemma 2.1,

$$
\left|m_{u}^{\gamma}(B)-u(x)\right|=\left|m_{u-u(x)}^{\gamma}(B)\right| \leq m_{|u-u(x)|}^{\min \{\gamma, 1-\gamma\}}(B),
$$

and the claim follows.

If we restrict the value of $p$ such that $\mathcal{F}$-functions are locally integrable, then Theorem 4.11 has a counterpart formulated in terms of integral averages. The proof of Theorem 4.14, which is similar to the proof of Theorem 4.11, will be omitted.

Theorem 4.14. Let $\mathcal{B}$ be a Busemann-Feller basis on a metric measure space $X$ satisfying (2.1). Let $\mathcal{F} \in\left\{N_{p, q}^{s}(X), M_{p, q}^{s}(X)\right\}$, where $0<s<1, Q /(Q+s)<p<\infty$, $0<q<\infty$, or $\mathcal{F}=M_{p, \infty}^{s}(X)=M^{s, p}(X)$, where $0<s \leq 1$ and $Q /(Q+s)<p<\infty$. Then the following claims are equivalent. 
(1) For every quasicontinuous $u \in \mathcal{F}$, there exists a set $E$ with $C_{\mathcal{F}}(E)=0$ such that

$$
\lim _{\mathcal{B} \ni B \rightarrow x} u_{B}=u(x)
$$

for every $x \in X \backslash E$.

(2) There exists a constant $C$ such that

$$
C_{\mathcal{F}}\left(\left\{x \in X: \limsup _{\mathcal{B} \ni B \rightarrow x}|u|_{B}>\lambda\right\}\right) \leq C \lambda^{-p}\|u\|_{\mathcal{F}}^{p}
$$

for every $u \in \mathcal{F}$.

(3) For each $\lambda>0$ and for each sequence $\left(u_{k}\right)$ such that $\left\|u_{k}\right\|_{\mathcal{F}} \rightarrow 0$ as $k \rightarrow \infty$, we have

$$
C_{\mathcal{F}}\left(\left\{x \in X: \limsup _{\mathcal{B} \ni B \rightarrow x}\left|u_{k}\right|_{B}>\lambda\right\}\right) \rightarrow 0
$$

as $k \rightarrow \infty$.

For the proof of our next result, Theorem 4.16, we need the following lemma. The proof given below is a modification of the proof of [21, Theorem 4.1]. We do not know whether the lemma holds true when $p \leq 1$ or $q \leq 1$.

Lemma 4.15. Let $0<s \leq 1,1<p<\infty, 1<q \leq \infty$ and $\mathcal{F} \in\left\{M_{p, q}^{s}(X), N_{p, q}^{s}(X)\right\}$. Then

$$
C_{\mathcal{F}}(U)=\lim _{i \rightarrow \infty} C_{\mathcal{F}}\left(U_{i}\right)
$$

whenever $U_{1} \subset U_{2} \subset \cdots$ are open subsets of $X$ and $U=\cup_{i=1}^{\infty} U_{i}$.

Proof. We prove the case $\mathcal{F}=M_{p, q}^{s}(X)$. The proof of the other case is similar. By monotonicity,

$$
\lim _{i \rightarrow \infty} C_{M_{p, q}^{s}(X)}\left(U_{i}\right) \leq C_{M_{p, q}^{s}(X)}(U) .
$$

To prove the opposite inequality, we may assume that $\lim _{i \rightarrow \infty} C_{M_{p, q}^{s}(X)}\left(U_{i}\right)<\infty$. Let $\varepsilon>0$ and let $u_{i} \in \mathcal{A}_{M_{p, q}^{s}(X)}^{\prime}\left(U_{i}\right)$ and $\bar{g}_{i}=\left(g_{k}^{i}\right)_{k \in \mathbb{Z}} \in \mathbb{D}^{s}\left(u_{i}\right)$ be such that

$$
\left(\left\|u_{i}\right\|_{L^{p}(X)}+\left\|\bar{g}_{i}\right\|_{L^{p}\left(X, l^{q}\right)}\right)^{p}<C_{M_{p, q}^{s}(X)}\left(U_{i}\right)+\varepsilon .
$$

Then $\left(u_{i}\right)$ is bounded in $L^{p}(X)$ and $\left(\bar{g}_{i}\right)$ is bounded in $L^{p}\left(X, l^{q}\right)$. Hence, by passing to a subsequence, we may assume that $u_{i} \rightarrow u$ weakly in $L^{p}(X)$ and $\bar{g}_{i} \rightarrow \bar{g}$ weakly in $L^{p}\left(X, l^{q}\right)$. Using Mazur's lemma, we obtain convex combinations $v_{j}=\sum_{i=j}^{n_{j}} \lambda_{j, i} u_{i}$ and $\bar{h}_{j}=\sum_{i=j}^{n_{j}} \lambda_{j, i} \bar{g}_{i}$ such that $v_{j} \rightarrow u$ in $L^{p}(X), \bar{h}_{j} \rightarrow \bar{g}$ in $L^{p}\left(X, l^{q}\right)$ as $j \rightarrow \infty$ and $\bar{h}_{j} \in \mathbb{D}^{s}\left(v_{j}\right)$. Passing to a subsequence, we may also assume that $v_{j} \rightarrow u$ and $\bar{h}_{j} \rightarrow \bar{g}$ pointwise almost everywhere as $j \rightarrow \infty$. This easily implies that $\bar{g} \in \mathbb{D}^{s}(u)$. Since $u_{i}=1$, it follows that, for every $x \in U, v_{j}(x)=1$ for $j$ large enough. Hence $u=1$ in $U$ and so $u \in \mathcal{A}_{M_{p, q}^{s}(X)}^{\prime}(U)$. By the weak lower semicontinuity of norms,

$$
\begin{aligned}
C_{M_{p, q}^{s}(X)}(U) & \leq\left(\|u\|_{L^{p}(X)}+\|\bar{g}\|_{L^{p}\left(X, l^{q}\right)}\right)^{p} \\
& \leq \liminf _{i \rightarrow \infty}\left(\left\|u_{i}\right\|_{L^{p}(X)}+\left\|\bar{g}_{i}\right\|_{L^{p}\left(X, l^{q}\right)}\right)^{p} \\
& \leq \lim _{i \rightarrow \infty} C_{M_{p, q}^{s}(X)}\left(U_{i}\right)+\varepsilon
\end{aligned}
$$

and the claim follows by letting $\varepsilon \rightarrow 0$. 
Theorem 4.16. Let $\mathcal{B}$ be a Busemann-Feller basis on a separable metric measure space $X$. Let $\mathcal{F} \in\left\{N_{p, q}^{s}(X), M_{p, q}^{s}(X)\right\}$, where $0<s<1,1<p, q<\infty$, or $\mathcal{F}=M_{p, \infty}^{s}(X)=M^{s, p}(X)$, where $0<s \leq 1$ and $1<p<\infty$. Consider the following claims.

(1) For each $0<\gamma<1$ and $u \in \mathcal{F}$,

$$
C_{\mathcal{F}}\left(\left\{x \in X: \mathcal{M}_{\mathcal{B}}^{\gamma} u(x)>\lambda\right\}\right) \rightarrow 0
$$

as $\lambda \rightarrow \infty$.

(2) For each $0<\gamma<1, \lambda>0$ and for each sequence $\left(u_{k}\right)$ such that $\left\|u_{k}\right\|_{\mathcal{F}} \rightarrow 0$ as $k \rightarrow \infty$, we have

$$
C_{\mathcal{F}}\left(\left\{x \in X: \mathcal{M}_{\mathcal{B}}^{\gamma} u_{k}(x)>\lambda\right\}\right) \rightarrow 0
$$

as $k \rightarrow \infty$.

(3) For every quasicontinuous $u \in \mathcal{F}$, there exists a set $E$ with $C_{\mathcal{F}}(E)=0$ such that

$$
\lim _{\mathcal{B} \ni B \rightarrow x} m_{u}^{\gamma}(B)=u(x)
$$

for every $0<\gamma<1$ and $x \in X \backslash E$.

Then $(1) \Longrightarrow(2) \Longrightarrow(3)$.

Implication $(2) \Longrightarrow(3)$ of Theorem 4.16 follows from Theorem 4.11 (because condition (2) of Theorem 4.16 trivially implies condition (3) of Theorem 4.11). Hence, it suffices to prove the following lemma.

Lemma 4.17. Suppose that the assumptions of Theorem 4.16 are in force. Let $0<\gamma<1$ and suppose that, for every $u \in \mathcal{F}$,

$$
C_{\mathcal{F}}\left(\left\{x \in X: \mathcal{M}_{\mathcal{B}}^{\gamma / 4} u(x)>\lambda\right\}\right) \rightarrow 0
$$

as $\lambda \rightarrow \infty$. Then, for every $\lambda>0$,

$$
C_{\mathcal{F}}\left(\left\{x \in X: \mathcal{M}_{\mathcal{B}}^{\gamma} u_{i}(x)>\lambda\right\}\right) \rightarrow 0
$$

as $\left\|u_{i}\right\|_{\mathcal{F}} \rightarrow 0$.

Proof. Denote by $Y$ the set of measurable functions for which

$$
\lim _{\lambda \rightarrow \infty} C_{\mathcal{F}}(\{x \in X:|u(x)|>\lambda\})=0 .
$$

and equip $Y$ with a functional

$$
\|u\|_{Y}=\inf _{\lambda>0}\left\{\lambda+C_{\mathcal{F}}(\{x \in X:|u(x)|>\lambda\})^{r}\right\},
$$

where $r$ is the exponent from (4.3). Then $\|u\|_{Y}=0$ if and only if $u=0 \mathcal{F}$ quasieverywhere. Moreover, there exists a constant $C \geq 1$ such that

$$
\|u+v\|_{Y} \leq C\left(\|u\|_{Y}+\|v\|_{Y}\right)
$$

for every $u, v \in Y$ and

$$
\left\|a_{n} u\right\|_{Y} \rightarrow 0 \text { whenever } u \in Y \text { and } a_{n} \rightarrow 0 \text {. }
$$


It is easy to see that

$$
\lim _{i \rightarrow \infty} C_{\mathcal{F}}\left(\left\{x \in X:\left|u_{i}(x)-u(x)\right|>\lambda\right\}\right)=0,
$$

for every $\lambda>0$, if and only if $\lim _{i \rightarrow \infty}\left\|u_{i}-u\right\|_{Y}=0$.

Since $\widetilde{d}_{Y}(u, v)=\|u-v\|_{Y}$ is a quasi-metric on $Y$, there exists a metric $d_{Y}$ on $Y$ and a constant $0<\alpha \leq 1$ such that

$$
d_{Y}(u, v) \leq\|u-v\|_{Y}^{\alpha} \leq 2 d_{Y}(u, v)
$$

for all $u, v \in Y$, see e.g. [12, Proposition 14.5].

Let $\mathcal{B}^{\prime}=\left\{B_{j}\right\}$ be the countable Busemann-Feller basis given by Lemma 3.9. Denote

$$
\mathcal{M}_{k}^{\gamma / 2} u=\max _{1 \leq j \leq k} m_{|u|}^{\gamma / 2}\left(B_{j}\right) \chi_{B_{j}} .
$$

Then operators $\mathcal{M}_{k}^{\gamma / 2}$ are continuous from $L^{0}(X)$ to $Y$ (and so from $\mathcal{F}$ to $Y$ ). Indeed, if $u_{i} \rightarrow u$ in $L^{0}(X)$, then by Lemma 3.7, for every $j, m_{\left|u_{i}\right|}^{\gamma / 2}\left(B_{j}\right) \rightarrow m_{|u|}^{\gamma / 2}\left(B_{j}\right)$ as $i \rightarrow \infty$. Since

$$
\left|\mathcal{M}_{k}^{\gamma / 2} u_{i}-\mathcal{M}_{k}^{\gamma / 2} u\right| \leq \max _{1 \leq j \leq k}\left|m_{\left|u_{i}\right|}^{\gamma / 2}\left(B_{j}\right)-m_{|u|}^{\gamma / 2}\left(B_{j}\right)\right| \chi_{B_{j}},
$$

it follows that, for every $\lambda>0$,

$$
C_{\mathcal{F}}\left(\left\{x \in X:\left|\mathcal{M}_{k}^{\gamma / 2} u_{i}(x)-\mathcal{M}_{k}^{\gamma / 2} u(x)\right|>\lambda\right\}\right)=0
$$

for large enough $i$, which in turn implies that $d_{Y}\left(\mathcal{M}_{k}^{\gamma / 2} u_{i}, \mathcal{M}_{k}^{\gamma / 2} u\right) \rightarrow 0$ as $i \rightarrow \infty$.

Let $\varepsilon>0$. For $n \in \mathbb{N}$, denote

$$
F_{n}=\left\{u \in \mathcal{F}: \sup _{k} d_{Y}\left(n^{-1} M_{k}^{\gamma / 2} u, 0\right) \leq \varepsilon\right\} .
$$

By continuity, sets $F_{n}$ are closed. Let $u \in \mathcal{F}$. Since, by assumption, $\mathcal{M}_{\mathcal{B}}^{\gamma / 4} u \in Y$, Lemma 3.9 implies that $\mathcal{M}_{\mathcal{B}^{\prime}}^{\gamma / 2} u \in Y$. Hence, by (4.6) and (4.5),

$$
\sup _{k} d_{Y}\left(n^{-1} M_{k}^{\gamma / 2} u, 0\right) \leq \sup _{k}\left\|n^{-1} M_{k}^{\gamma / 2} u\right\|_{Y}^{\alpha} \leq\left\|n^{-1} M_{\mathcal{B}^{\prime}}^{\gamma / 2} u\right\|_{Y}^{\alpha} \rightarrow 0
$$

as $n \rightarrow \infty$. It follows that $\mathcal{F}=\cup_{n=1}^{\infty} F_{n}$. By the Baire-Hausdorff theorem ([32, p.11]), one of the sets $F_{n}$ must have non-empty interior. Thus, there is $n_{0} \in \mathbb{N}$, $u_{0} \in F_{n_{0}}$ and $\delta>0$ such that $B_{\mathcal{F}}\left(u_{0}, \delta\right) \subset F_{n_{0}}$. Now, if $\|u\|_{\mathcal{F}}<\delta$, then $u+u_{0} \in F_{n_{0}}$, and so

$$
\left\|\mathcal{M}_{k}^{\gamma} n_{0}^{-1} u\right\|_{Y} \leq C\left(\left\|n_{0}^{-1} \mathcal{M}_{k}^{\gamma / 2}\left(u_{0}+u\right)\right\|_{Y}+\left\|n_{0}^{-1} \mathcal{M}_{k}^{\gamma / 2} u_{0}\right\|_{Y}\right) \leq C \varepsilon
$$

for every $k \in \mathbb{N}$. It follows that $\sup _{k}\left\|\mathcal{M}_{k}^{\gamma} u_{i}\right\|_{Y} \rightarrow 0$ as $\left\|u_{i}\right\|_{\mathcal{F}} \rightarrow 0$. This, Lemma 3.9 and Lemma 4.15 imply that, for every $\lambda>0$,

$$
\begin{aligned}
C_{\mathcal{F}}\left(\left\{x \in X: \mathcal{M}_{\mathcal{B}}^{\gamma} u_{i}(x)>\lambda\right\}\right) & \leq C_{\mathcal{F}}\left(\left\{x \in X: \mathcal{M}_{\mathcal{B}^{\prime}}^{\gamma} u_{i}(x)>\lambda\right\}\right) \\
& =\sup _{k} C_{\mathcal{F}}\left(\left\{x \in X: \mathcal{M}_{k}^{\gamma} u_{i}(x)>\lambda\right\}\right) \rightarrow 0
\end{aligned}
$$

as $\left\|u_{i}\right\|_{\mathcal{F}} \rightarrow 0$.

A similar reasoning as above gives the following result for the usual maximal function. The proof of the theorem will be omitted. 
Theorem 4.18. Let $\mathcal{B}$ be a Busemann-Feller basis on a separable metric measure space $X$. Let $\mathcal{F} \in\left\{N_{p, q}^{s}(X), M_{p, q}^{s}(X)\right\}$, where $0<s<1,1<p, q<\infty$, or $\mathcal{F}=M_{p, \infty}^{s}(X)=M^{s, p}(X)$, where $0<s \leq 1$ and $1<p<\infty$. Consider the following claims.

(1) For each $u \in \mathcal{F}$,

$$
C_{\mathcal{F}}\left(\left\{x \in X: \mathcal{M}_{\mathcal{B}} u(x)>\lambda\right\}\right) \rightarrow 0
$$

as $\lambda \rightarrow \infty$.

(2) For each $\lambda>0$ and for each sequence $\left(u_{k}\right)$ such that $\left\|u_{k}\right\|_{\mathcal{F}} \rightarrow 0$ as $k \rightarrow \infty$, we have

$$
C_{\mathcal{F}}\left(\left\{x \in X: \mathcal{M}_{\mathcal{B}} u_{k}(x)>\lambda\right\}\right) \rightarrow 0
$$

as $k \rightarrow \infty$.

(3) For every quasicontinuous $u \in \mathcal{F}$, there exists a set $E$ with $C_{\mathcal{F}}(E)=0$ such that

$$
\lim _{\mathcal{B} \ni B \rightarrow x} u_{B}=u(x)
$$

for every $x \in X \backslash E$.

Then $(1) \Longrightarrow(2) \Longrightarrow(3)$.

\section{REFERENCES}

[1] M. de Guzmán: Differentiation of integrals in $\mathbb{R}^{n}$, Springer-Verlag, LNM 481, BerlinHeidelberg-New York, 1975.

[2] M. de Guzmán: Real-variable methods in Fourier analysis, North-Holland Publishing co., Amsterdam, 1981.

[3] H. Federer and W.P. Ziemer: The Lebesgue set of a function whose distribution derivatives are $p$-th power summable, Indiana Univ. Math. J. 22 (1972), 139-158.

[4] N. Fujii: A condition for a two-weight norm inequality for singular integral operators, Studia Math. 98 (1991), no. 3, 175-190.

[5] A. Gogatishvili, P. Koskela and Y. Zhou: Characterizations of Besov and Triebel-Lizorkin Spaces on Metric Measure Spaces, Forum Math. 25 (2013), no. 4, 787-819.

[6] P. Hajłasz: Sobolev spaces on an arbitrary metric space, Potential Anal. 5 (1996), 403-415.

[7] P. Harjulehto and J. Kinnunen: Differentiation bases for Sobolev functions on metric spaces, Publ. Mat. 48 (2004), no. 2, 381-395.

[8] T. Heikkinen, L. Ihnatsyeva and H. Tuominen: Measure density and extension of Besov and Triebel-Lizorkin functions, J. Fourier Anal. Appl. 22 (2016), no. 2, 334-382.

[9] T. Heikkinen, P. Koskela and H. Tuominen: Approximation and quasicontinuity of Besov and Triebel-Lizorkin functions, Trans. Amer. Math. Soc. 369 (2017), 3547-3573.

[10] T. Heikkinen and H. Tuominen: Smoothing properties of the discrete fractional maximal operator on Besov and Triebel-Lizorkin spaces, Publ. Mat. 58 (2014), no. 2. 379-399.

[11] T. Heikkinen and H. Tuominen: Approximation by Hölder functions in Besov and TriebelLizorkin spaces, Constr. Approx. 44 (2016), no. 3, 455-482.

[12] J. Heinonen: Lectures on analysis on metric spaces, Universitext, Springer-Verlag, New York, 2001.

[13] T. Hytönen: The A2 theorem: remarks and complements. In Harmonic analysis and partial differential equations, volume 612 of Contemp. Math., pages 91-106. Amer. Math. Soc., Providence, RI, 2014.

[14] T. Hänninen: Remark on dyadic pointwise domination and median oscillation decomposition, Houston J. Math. 43 (2017), no. 1, p. 183-197. 
[15] B. Jawerth, C. Perez, and G. Welland: The positive cone in Triebel-Lizorkin spaces and the relation among potential and maximal operators, Harmonic analysis and partial differential equations (Boca Raton, FL, 1988), 71-91, Contemp. Math., 107, Amer. Math. Soc., Providence, RI, 1990.

[16] B. Jawerth and A. Torchinsky: Local sharp maximal functions, J. Approx. Theory 43 (1985), no. $3,231-270$.

[17] F. John: Quasi-isometric mappings, Seminari 1962/63 Anal. Alg. Geom. e Topol., vol. 2, Ist. Naz. Alta Mat., Ediz. Cremonese, Rome, 1965, pp. 462-473.

[18] N. Karak: Generalized Lebesgue points for Sobolev functions, Czech Math J 67 (2017), 143-150. doi:10.21136/CMJ.2017.0405-15

[19] T. Kilpeläinen: On the uniqueness of quasicontinuous functions, Ann. Acad. Sci. Fenn. Math. 23 (1998), 261-262.

[20] T. Kilpeläinen, J. Kinnunen, O. Martio: Sobolev Spaces with Zero Boundary Values on Metric Spaces, Potential Anal. 12 (2000), 233-247.

[21] J. Kinnunen and O. Martio. Choquet property for the Sobolev capacity in metric spaces. In Proceedings of the conference on Analysis and Geometry held in Novosibirsk, 285-290, 2000.

[22] P. Koskela and E. Saksman: Pointwise characterizations of Hardy-Sobolev functions, Math. Res. Lett. 15 (2008), no. 4, 727-744.

[23] P. Koskela, D. Yang and Y. Zhou: Pointwise Characterizations of Besov and Triebel-Lizorkin Spaces and Quasiconformal Mappings, Adv. Math. 226 (2011), no. 4, 3579-3621.

[24] A. K. Lerner: A pointwise estimate for the local sharp maximal function with applications to singular integrals, Bull. London Math. Soc. 42 (2010), 843-856.

[25] A. K. Lerner: On an estimate of Calderón-Zygmund operators by dyadic positive operators. J. Anal. Math. 121, 141-161, 2013.

[26] A. K. Lerner and C. Pérez: Self-improving properties of generalized Poincaré type inequalities throught rearrangements, Math. Scand. 97 (2) (2005), 217-234.

[27] A. K. Lerner and C. Pérez: A New Characterization of the Muckenhoupt $A_{p}$ Weights Through an Extension of the Lorentz-Shimogaki Theorem, Indiana math. J. 56, no 6 (2007), 26972722.

[28] J. Poelhuis and A. Torchinsky: Medians, continuity, and vanishing oscillation, Studia Math. 213 (2012), no. 3, 227-242.

[29] N. Shanmugalingam, D. Yang, and W. Yuan: Newton-Besov Spaces and NewtonTriebel-Lizorkin Spaces on Metric Measure Spaces, to appear in Positivity, http://dx.doi.org/10.1007/s11117-014-0291-7

[30] J-O. Strömberg: Bounded mean oscillation with Orlicz norms and duality of Hardy spaces, Indiana Univ. Math. J. 28 (1979), no. 3, 511-544.

[31] J-O. Strömberg and A. Torchinsky: Weighted Hardy spaces. Lecture Notes in Mathematics, 1381. Springer-Verlag, Berlin, 1989.

[32] D. Yang: New characterizations of Hajłasz-Sobolev spaces on metric spaces, Sci. China Ser. A 46 (2003), 675-689.

[33] Y. Zhou: Fractional Sobolev extension and imbedding, Trans. Amer. Math. Soc. 367 (2015), no. 2, 959-979.

Department of Mathematics, Aalto University, P.O. Box 11100, Fi-00076 Aalto UNIVERSITY, FinlAND

E-mail address: toni.heikkinen@aalto.fi,juha.k.kinnunen@aalto.fi 